\title{
Coffee consumption and incidence of impaired fasting glucose, impaired glucose tolerance, and type 2 diabetes: the Hoorn Study
}

\author{
R. M. van Dam ${ }^{1}$ - J. M. Dekker ${ }^{2}$ G. Nijpels ${ }^{2}$ C. D. A. Stehouwer ${ }^{2,3}$ • L. M. Bouter ${ }^{2}$ R. J. Heine ${ }^{2,4}$ \\ ${ }^{1}$ Department of Nutrition and Health, Faculty of Earth and Life Sciences, Vrije University Amsterdam, Amsterdam, \\ The Netherlands \\ 2 Institute for Research in Extramural Medicine, Vrije University Medical Centre, Amsterdam, The Netherlands \\ ${ }^{3}$ Department of Internal Medicine, Vrije University Medical Centre, Amsterdam, The Netherlands \\ ${ }^{4}$ Department of Endocrinology, Vrije University Medical Centre, Amsterdam, The Netherlands
}

\section{Abstract}

Aims/hypothesis. Coffee contains several substances that may affect glucose metabolism. The aim of this study was to evaluate the relationship between habitual coffee consumption and the incidence of IFG, IGT and type 2 diabetes.

Methods. We used cross-sectional and prospective data from the population-based Hoorn Study, which included Dutch men and women aged 50-74 years. An OGTT was performed at baseline and after a mean follow-up period of 6.4 years. Associations were adjusted for potential confounders including BMI, cigarette smoking, physical activity, alcohol consumption and dietary factors.

Results. At baseline, a 5 cup per day higher coffee consumption was significantly associated with lower fasting insulin concentrations $(-5.6 \%, 95 \% \mathrm{CI}-9.3$ to $-1.6 \%)$ and $2-\mathrm{h}$ glucose concentrations $(-8.8 \%, 95 \%$ CI -11.8 to $-5.6 \%$ ), but was not associated with lower fasting glucose concentrations $(-0.8 \%, 95 \%$ CI -2.1 to $0.6 \%$ ). In the prospective analyses, the odds ratio (OR) for IGT was 0.59 (95\% CI 0.36-0.97) for 3-4 cups per day, 0.46 (95\% CI $0.26-0.81)$ for 5-6 cups per day, and 0.37 (95\% CI $0.16-0.84$ ) for 7 or more cups per day, as compared with the corresponding values for the consumption of 2 or fewer cups of coffee per day ( $p=0.001$ for trend). Higher coffee consumption also tended to be associated with a lower incidence of type 2 diabetes (OR 0.69, CI 0.31-1.51 for $\geq 7$ vs $\leq 2$ cups per day, $p=0.09$ for trend), but was not associated with the incidence of IFG (OR 1.35, CI 0.80-2.27 for $\geq 7$ vs $\leq 2$ cups per day, $p=0.49$ for trend).

Conclusions/interpretation. Our findings indicate that habitual coffee consumption can reduce the risk of IGT, and affects post-load rather than fasting glucose metabolism.

Keywords Coffee $\cdot$ IFG · IGT · Insulin · Prospective study · Type 2 diabetes.
Received: 13 April 2004 / Accepted: 5 August 2004

Published online: 11 December 2004

(C) Springer-Verlag 2004

R. M. van Dam (๘)

Department of Nutrition and Health,

Faculty of Earth and Life Sciences,

Vrije University Amsterdam, de Boelelaan 1085,

1081 HV Amsterdam, The Netherlands

E-mail: rob.van.dam@falw.vu.nl

Fax: +31-20-4446940

Abbreviations: HOMA-IR, homeostasis model assessment index for insulin resistance $\cdot$ OR, odds ratio

\section{Introduction}

Coffee is among the most widely consumed beverages in the world. Various potential health effects of coffee have been extensively studied $[1,2,3,4,5,6]$, but data on habitual coffee consumption and the development of type 2 diabetes have only recently been published $[7,8,9,10,11,12,13]$. Coffee contains several substances that affect glucose metabolism. Coffee is a major source of caffeine and chlorogenic acid [14], and contains substantial amounts of magnesium and other micronutrients. Chlorogenic acid is a phenol that may reduce hepatic glucose output through inhibition of glucose-6-phosphate hydrolysis $[15,16]$. Roasting 
of coffee can transform some of the chlorogenic acid into quinides, which have been shown to enhance insulin action in rats [17]. Caffeine acutely lowered insulin sensitivity $[18,19,20,21,22]$ and increased glucose concentrations [23, 24, 25, 26, 27] in short-term metabolic studies in humans. However, these effects may not persist during chronic coffee consumption [19], and beneficial effects of caffeine on insulin sensitivity [28] and glucose-induced insulin secretion [29] have also been suggested. In several cohort studies, magnesium intake was associated with a lower risk of type 2 diabetes [30,31,32], and in intervention studies, pharmacological doses of magnesium improved glucose metabolism [33].

Knowledge of both the adverse and beneficial health effects of coffee consumption is necessary to permit prioritisation of public health initiatives and to allow individuals to make informed choices regarding coffee consumption. In addition, information on the possible beneficial health effects of specific constituent compounds in coffee may lead to the development of coffees or other products with increased contents of these substances. Dutch [7], US [11], Swedish [10] and Finnish [12,13] cohort studies have reported that higher coffee consumption is associated with a lower risk of type 2 diabetes. Because these studies only included diagnosed diabetes as an endpoint, they provided little insight into the pathways through which coffee consumption may affect the development of type 2 diabetes. In addition, it has been suggested that detection bias may have affected the results of studies on coffee and clinical type 2 diabetes [9]. We therefore examined the relationship between coffee consumption and the incidence of IFG, IGT and type 2 diabetes, as assessed by repeated OGTTs, in the Hoorn Study. We also investigated possible cross-sectional associations between coffee consumption and different markers of insulin sensitivity and glucose metabolism.

\section{Subjects, materials and methods}

Study population and design. The Hoorn Study is a populationbased cohort study on glucose intolerance in older Dutch men and women [34]. Baseline data were collected from October 1989 through December 1991. Participants were men and women aged 50-74 years who were randomly selected from the municipal registry of the medium-sized town of Hoorn. The participation rate was $71 \%$ and, after exclusion of nonCaucasians $(n=56)$ and persons with known diabetes $(n=90)$, 2394 participants remained. We excluded participants with missing information for fasting glucose concentrations $(n=4)$, coffee consumption or any of the examined potential confounders $(n=112)$, leaving 2280 persons in the baseline analyses.

Between January 1996 and December 1998, 2086 participants were invited to attend a follow-up examination. The other members of the original cohort were not invited for logistic reasons $(n=140)$, or because they had died $(n=150)$ or had moved out of Hoorn $(n=108)$. A total of 1513 persons $(72.5 \%)$ participated in the follow-up measurements, and we obtained complete information on glucose tolerance status at follow-up for 1479 of these subjects. We excluded those who had known or newly detected diabetes at baseline $(n=132)$, or missing information on coffee consumption or any of the examined potential confounders $(n=38)$. As a result, the present prospective analysis included 1312 participants. The average time between the baseline and the follow-up measurement was 6.4 years. The Hoorn Study was approved by the Ethical Review Committee of the Vrije University Medical Centre and informed consent was obtained from all participants.

Assessment of outcome parameters. Oral glucose tolerance tests (OGTT) were performed between 8.00 and 10.00 hours. Participants were instructed to abstain from alcohol from 17.00 hours and to fast (except for drinking water) from 22.00 hours on the previous evening. If instructions had not been followed, or in case of fever or an unusual physical activity pattern the previous 3 days, the OGTT was postponed. Blood samples were collected before and $2 \mathrm{~h}$ after a glucose load consisting of $75 \mathrm{~g}$ glucose anhydrate in $300 \mathrm{ml}$ of water ingested over the course of $5 \mathrm{~min}$. Laboratory analyses were conducted at the Vrije University Medical Centre. Serum proinsulin and 2-h insulin concentrations were only determined in a random sample taken within strata for sex, age and glucose tolerance [34]. Plasma glucose was determined directly, and serum was stored at $-20^{\circ} \mathrm{C}$ for the determination of insulin and proinsulin concentrations. Glucose was measured using a glucose dehydrogenase method (Merck, Darmstadt, Germany). Immuno-specific insulin was measured in serum by a double-antibody RIA (antibody SP21; Linco Research, St. Louis, Mo., USA). Proinsulin was measured by a double-antibody RIA, based on reagents from R. Bowsher (Lilly Laboratory for Clinical Research, Indianapolis, Ind., USA). The inter-assay analytical CV was $1.4 \%$ for glucose and $6 \%$ for insulin levels within the range of 40-1000 pmol/1 (lower limit of sensitivity $12 \mathrm{pmol} / \mathrm{l}$ ), and 6\% (at $100 \mathrm{pmol} / \mathrm{l}$ ) to $15 \%$ (at lower levels) for proinsulin (lower limit of sensitivity $3 \mathrm{pmol} / \mathrm{l}$ ). Fasting glucose and insulin concentrations were used to calculate the homeostasis model assessment index for insulin resistance (HOMA-IR) as described by Matthews et al. [35]. Normal fasting glucose, normal glucose tolerance, IFG, IGT and diabetes were defined according to the 1999 criteria of the World Health Organization [36].

Assessment of coffee consumption and potential confounders. We used a 92-item semi-quantitative food-frequency questionnaire to assess average food intake, including alcohol and coffee consumption. This instrument included the questions "How often do you usually drink coffee?" (11 answer categories) and "How much coffee do you drink on average on such a day?" (where the answer was provided in terms of cups consumed and, according to the Dutch definition, one cup is equivalent to $125 \mathrm{ml}$ ). In addition, specific questions were asked about addition of milk or cream to coffee, and the addition of sugar (or honey or syrup) to coffee, tea, yoghurt or curds, porridge, and breakfast cereals. The questionnaire was completed by the participants at home and was subsequently checked during an interview at the research centre. Nutrient intake was calculated using a computerised version of the Dutch Food Composition Table [37]. This questionnaire was validated by comparison with a dietary history interview that was conducted in 74 male and female inhabitants of Hoorn at home by a dietician [37]. Pearson correlation coefficients for estimates from the questionnaire and the dietary history were 0.73 for saturated fat $(\mathrm{g}), 0.68$ for polyunsaturated fat $(\mathrm{g})$, and 0.70 for fibre intake $(\mathrm{g})$. 
Physical activity was assessed by the same questionnaire as used in the Zutphen Elderly Study, with slight modifications [38]. A score was calculated (1 point per activity, range 0-9) based on involvement (yes or no) in sports, cycling, gardening, walking, odd jobs, climbing staircases at home, household activities, work and daily food shopping. Information on history of cardiovascular diseases was obtained using a questionnaire based on the Rose questionnaire [39]. Cigarette smoking habits and medication use were also assessed by questionnaire. Height and weight were measured without shoes and outer garments, and BMI was calculated as weight $(\mathrm{kg})$ divided by height squared $\left(\mathrm{m}^{2}\right)$. Waist circumference was measured at the level midway between the lowest rib margin and the iliac crest, and hip circumference was measured at the widest level over the greater trochanters. WHR was calculated as waist circumference divided by hip circumference.

Statistical analysis. Statistical analyses were carried out using SAS software (SAS Institute, Cary, N.C., USA). We used multivariate linear regression analysis to examine associations between coffee consumption and markers of glucose metabolism and insulin sensitivity at baseline. As outcome variables were not normally distributed (except for 2-h glucose), we logarithmically transformed these variables to correspond with the assumptions for regression analysis. Regression coefficients were re-transformed and expressed as percentage difference in dependent variables for a defined difference in coffee consumption ( 5 cups per day: the difference between the 10th and 90th percentile of coffee consumption). We calculated adjusted differences in 2-h glucose concentrations according to categories of coffee consumption using analysis of covariance.

For the prospective data, we performed logistic regression analysis to calculate odds ratios (ORs) and 95\% confidence intervals. The analyses included participants without diabetes at baseline for incidence of type 2 diabetes $(n=1312)$, participants with normal glucose tolerance at baseline and without diabetes at follow-up for incidence of IGT $(n=1117)$, and participants with normal fasting glucose at baseline and without diabetes at follow-up for incidence of IFG $(n=1116)$. We used the same categories of coffee consumption as those defined in a previous study on coffee consumption and type 2 diabetes [7]. We combined the upper two categories for analyses stratified by sex to avoid imprecise estimates due to low numbers. The multivariate model included covariables that were entered as continuous variables (age, BMI, WHR, physical activity score, and intake of total energy, fibre, saturated fat and polyunsaturated fat), and variables that were entered as dichotomous dummy variables for each category except for the reference category (sex, alcohol consumption [none, $\leq 10,10-30$ or $>30 \mathrm{~g} / \mathrm{day}$ ], cigarette smoking [never, past or current], history of cardiovascular diseases [yes or no] and use of antihypertensive medication [yes or no]). We conducted additional analyses with adjustment for added sugar intake (g/day) and magnesium intake (mg/day). Magnesium intake was not adjusted to correct for confounding, but rather to examine whether the magnesium supplied by coffee explained the association between coffee consumption and glucose tolerance. We also examined associations between tea consumption and parameters of glucose metabolism, because tea is also a source of caffeine and phenolic compounds with antioxidant activity. Saturated and polyunsaturated fat intakes were expressed as percentages of total energy intake. Fibre and magnesium intakes were adjusted for total energy intake using the residual method [40]. The $p$ values for trend were calculated by modelling coffee consumption as a continuous variable. All $p$ values were two-sided, and a $p$ value less than 0.05 was considered statistically significant.

\section{Results}

Baseline data. The median habitual coffee consumption of this population was 4 cups per day (interquartile range 3-6 cups per day). Persons with a higher coffee consumption were more likely to be men and to smoke, and were less likely to use antihypertensive medication and to drink tea (Table 1). In addition, higher coffee consumption was associated with lower age and fibre intake, and higher alcohol and magnesium intake. Of the coffee drinkers, $43.7 \%$ used sugar in their coffee.

Post-load plasma glucose concentrations decreased with increasing levels of coffee consumption in a stepwise manner, and adjustment for potential confounders did not materially change this association (Table 2). Compared with the consumption of two cups of coffee or fewer per day, lower multivariate-adjusted 2-h post-load glucose concentrations were observed for the consumption of 3-4 cups per day $(-0.52 \mathrm{mmol}, 95 \% \mathrm{CI}-0.81$ to $-0.23 \mathrm{mmol} / \mathrm{l}), 4-5$ cups per day $(-0.76 \mathrm{mmol} / \mathrm{l}, 95 \%$ CI -1.07 to $-0.43 \mathrm{mmol} / \mathrm{l}$ ) and 7 cups or more per day $(-0.87 \mathrm{mmol} / 1,95 \% \mathrm{CI}-1.29$ to $-0.45 \mathrm{mmol} / \mathrm{l})$. When coffee consumption was analysed as a continuous variable using a multivariate model, a 5 cup per day higher consumption was associated with an $8.8 \%$ reduction in 2-h glucose concentration (Table 2). This inverse association remained essentially the same after further adjustment for magnesium intake $(-9.3 \%$ per additional 5 cups per day) or added sugar intake $(-8.4 \%$ per additional 5 cups per day). We examined the robustness of the association between coffee consumption and 2-h glucose concentrations in analyses excluding persons with cardiovascular diseases or using antihypertensive medication, coffee abstainers, users or non-users of milk in coffee, and users or nonusers of sugar in coffee, and by analyses within strata by sex, age $(<60$ or $\geq 60$ years), overweight $(<25$ or $\geq 25 \mathrm{~kg} / \mathrm{m}^{2}$ ), current smoking (yes or no), physical activity (score $<6$ or $\geq 6$ ), and alcohol consumption (yes or no). The inverse association between coffee consumption and 2-h glucose concentrations remained in all these analyses (all $p<0.05$, range -6.0 to $-11.0 \%$ per additional 5 cups per day).

We also examined whether coffee consumption was associated with other markers of insulin sensitivity and glucose metabolism (Table 2). Coffee consumption was significantly inversely associated with fasting and 2-h insulin concentrations and HOMA-IR values, whereas the inverse associations between coffee consumption and fasting glucose and proinsulin concentrations were not statistically significant. Tea consumption was inversely associated with fasting glucose concentrations $(-0.5 \%, 95 \% \mathrm{CI}-0.8$ to $-0.2 \%$ per cup per day, adjusted for coffee and the variables included in the multivariate model used for the coffee analysis), but was not significantly associated with 
Table 1. Baseline characteristics of the Hoorn Study population according to coffee consumption

\begin{tabular}{|c|c|c|c|c|}
\hline & \multicolumn{4}{|c|}{ Coffee consumption (cups per day) } \\
\hline & $\begin{array}{l}\leq 2 \\
(n=487)\end{array}$ & $\begin{array}{l}3-4 \\
(n=897)\end{array}$ & $\begin{array}{l}5-6 \\
(n=652)\end{array}$ & $\begin{array}{l}\geq 7 \\
(n=244)\end{array}$ \\
\hline Women & 63 & 58 & 49 & 40 \\
\hline Age (years) & $63.6(7.56)$ & $62.2(7.24)$ & $60.0(6.68)$ & $58.0(5.97)$ \\
\hline BMI $\left(\mathrm{kg} / \mathrm{m}^{2}\right)$ & $26.4(3.74)$ & $26.6(3.52)$ & $26.4(3.40)$ & $26.3(3.19)$ \\
\hline Men & $0.96(0.07)$ & $0.95(0.07)$ & $0.94(0.06)$ & $0.94(0.06)$ \\
\hline Women & $0.85(0.07)$ & $0.85(0.07)$ & $0.84(0.07)$ & $0.84(0.08)$ \\
\hline Physical activity score ${ }^{a}$ & $5.5(1.6)$ & $5.7(1.6)$ & $5.6(1.5)$ & $5.7(1.5)$ \\
\hline Cigarette smoking & 21 & 23 & 41 & 55 \\
\hline Polyunsaturated fat intake (\% energy) & $7.9(3.3)$ & $8.0(3.3)$ & $7.9(3.1)$ & $7.7(3.2)$ \\
\hline Fibre intake (g/day) & $27.0(6.9)$ & $27.2(6.2)$ & $26.4(5.9)$ & $25.2(5.6)$ \\
\hline Magnesium intake (mg/day) & $272(59)$ & $284(54)$ & $292(51)$ & $306(51)$ \\
\hline Tea drinkers & 95 & 92 & 80 & 59 \\
\hline Added sugar intake (g/day) & $5.0(0.0-17.0)$ & $5.0(0.0-24.0)$ & $9.0(0.0-36.0)$ & $6.0(0.0-45.5)$ \\
\hline Use of antihypertensive medication & 23 & 22 & 15 & 12 \\
\hline Cardiovascular disease & 19 & 19 & 17 & 19 \\
\hline
\end{tabular}

Values are medians (interquartile ranges) percentages or means (SD). a range 0-9

Table 2. Baseline data on differences in markers of hyperglycaemia and insulin resistance for an increment in coffee consumption of 5 cups per day in the Hoorn Study

\begin{tabular}{lcc}
\hline Marker & Age-and sex-adjusted modela & Multivariate-adjusted model $^{\mathrm{b}}$ \\
\hline 2-h glucose & $-9.9 \%(-13.0$ to $-6.8 \%)$ & $-8.8 \%(-11.8$ to $-5.6 \%)$ \\
Fasting glucose & $-0.8 \%(-2.1$ to $0.6 \%)$ & $-0.8 \%(-2.1$ to $0.6 \%)$ \\
2-h insulin & $-21.3 \%(-32.3$ to $-8.5 \%)$ & $-19.7 \%(-30.2$ to $-8.1 \%)$ \\
Fasting insulin & $-5.3 \%(-9.2$ to $-1.1 \%)$ & $-5.6 \%(-9.3$ to $-1.6 \%)$ \\
HOMA-IR & $-6.0 \%(-10.4$ to $-1.4 \%)$ & $-6.3 \%(-10.4$ to $-2.1 \%)$ \\
Fasting proinsulin & $-11.8 \%(-26.3$ to $5.6 \%)$ & $-13.1 \%(-26.9$ to $3.3 \%)$ \\
\hline
\end{tabular}

Values shown are percentage differences $(95 \% \mathrm{CI})$ per additional 5 cups per day. Data were complete for fasting glucose $(n=2280)$. Due to missing values, data are based on $n=2270$ for 2-h glucose, and $n=2229$ for fasting insulin and HOMA-IR. Due to sampling and missing values, data are based on $n=589$ for 2-h insulin, and $n=465$ for proinsulin. a Adjusted for age (years) and sex; ${ }^{b}$ adjusted for age (years), sex, BMI $\left(\mathrm{kg} / \mathrm{m}^{2}\right)$,

any of the other parameters presented in Table 2 (data not shown).

Prospective data. As shown in Table 3, higher coffee consumption was associated with a lower incidence of IGT (incidence $17.2 \%$ for $\leq 2$ cups per day, $6.5 \%$ for $\geq 7$ cups per day). This inverse association remained after multivariate adjustment for potential confounders (OR $0.37,95 \%$ CI $0.16-0.84$ for $\geq 7$ vs $\leq 2$ cups per day, $p=0.001$ for trend), and was similar for men and women (OR 0.44 for men, 0.42 for
WHR, physical activity (score), alcohol consumption (none, $\leq 10,10-30$ or $>30 \mathrm{~g} / \mathrm{day}$ ), cigarette smoking (never, past or current), history of cardiovascular disease (yes or no), use of antihypertensive medication (yes or no), and intake of fibre (g/day), total energy ( $\mathrm{kJ} /$ day), saturated fat (\% energy) and polyunsaturated fat (\% energy)

women for $\geq 5$ cups per day vs $\leq 2$ cups per day). Higher coffee consumption also tended to be associated with a lower incidence of type 2 diabetes (OR $0.69,95 \%$ CI $0.31-1.51$ for $\geq 7$ vs $\leq 2$ cups per day, $p=0.09$ for trend), but was not associated with the incidence of IFG. Additional adjustment for magnesium intake (IGT: OR $0.38,95 \%$ CI $0.16-0.90$ for $\geq 7$ vs $\leq 2$ cups per day) or added sugar intake (IGT: OR $0.33,95 \%$ CI $0.14-0.78$ for $\geq 7$ vs $\leq 2$ cups per day) did not materially alter the observed associations. Tea consumption was not significantly associated 
Table 3. Incidence of type 2 diabetes IGT and IFG according to coffee consumption in the Hoorn Study

Coffee consumption (cups per day)

$\leq 2 \quad 3-4 \quad 5-6 \quad \geq 7 \quad p$ value for trend

Type 2 diabetes

Cases

Age- and sex-adjusted OR

$11.1 \%(29 / 261)$

1 (referent)

$10.3 \%(52 / 506)$

1 (referent)

$0.95(0.59-1.55)$

$0.94(0.56-1.55)$

$9.2 \%(36 / 392)$
$0.91(0.54-1.55)$
$0.92(0.53-1.61)$

$7.2 \%(11 / 153)$

$0.76(0.36-1.59)$

$0.69(0.31-1.51)$

0.11

0.09

IGT

Cases

Age- and sex-adjusted OR

Multivariate $\mathrm{OR}^{\mathrm{a}}$

$17.2 \%(35 / 203)$
1 (referent)
1 (referent)

$10.7 \%(46 / 432)$
$0.60(0.37-0.96)$
$0.59(0.36-0.97)$

$8.2 \%(28 / 343)$

$0.50(0.29-0.87)$

$0.46(0.26-0.81)$

$6.5 \%(9 / 139)$

$0.44(0.20-0.96)$

$0.37(0.16-0.84)$

0.005

0.001

IFG

Cases

Age- and sex-adjusted OR

Multivariate $\mathrm{OR}^{\mathrm{a}}$

\author{
$24.1 \%(53 / 220)$ \\ 1 (referent) \\ 1 (referent)
}

$27.1 \%(115 / 425)$
$1.18(0.81-1.73)$
$1.18(0.80-1.75)$

\author{
$31.3 \%(42 / 134)$ \\ $1.42(0.87-2.32)$ \\ $1.35(0.80-2.27)$
}

0.31

0.49
Values shown for cases are $\%(n / n$ total). The analysis included $n=1312$ without diabetes at baseline for incidence of Type 2 diabetes, $n=1117$ with normal glucose tolerance at baseline and without diabetes at follow-up for incidence of IGT, and $n=1116$ with normal fasting glucose at baseline and without diabetes at follow-up for incidence of IFG. a OR adjusted for age (years), sex, BMI $\left(\mathrm{kg} / \mathrm{m}^{2}\right)$, WHR, physical activity (score), alcohol consumption (none, $\leq 10,10-30$ or $>30$ g/day), cigarette smoking (never, past or current), history of cardiovascular disease (yes or no), use of antihypertensive medication (yes or no), and intake of fibre ( $\mathrm{g} /$ day), total energy $(\mathrm{kJ} /$ day $)$, saturated fat $(\%$ energy) and polyunsaturated fat (\% energy) with any of the three studied endpoints (data not shown).

\section{Discussion}

In this population-based study of older men and women, higher habitual coffee consumption was associated with lower 2-h post-load glucose concentrations and a lower incidence of IGT. Coffee consumption also tended to be associated with a lower incidence of type 2 diabetes, but was not associated with fasting glucose concentrations or the incidence of IFG. These data suggest that coffee consumption affects post-load rather than fasting glucose metabolism. Coffee consumption was also cross-sectionally associated with lower values for markers of insulin resistance. Magnesium intake did not explain the observed associations.

Previous studies that reported an inverse association between coffee consumption and incidence of type 2 diabetes only included diagnosed cases [7, 10, $11,12,13]$. The present study included repeated OGTTs in all participants; thus, detection bias can be excluded as a possible explanation for the observed associations. Misclassification of coffee consumption is unlikely to have affected the findings, as coffee use tends to be a constant and well-reported habit [7]. Although the Hoorn Study collected extensive information on potential confounders, residual confounding may have affected the results. However, the consistency of results across strata by risk factors and across cohorts in different countries argues against residual confounding as an explanation for the observed asso- ciations. Loss to follow-up can bias the results of prospective studies if it is associated with both the exposure and the outcome of interest. However, coffee consumption at baseline was similar for both those who did and did not participate in the follow-up measurements (both groups: median 4.0 cups per day, interquartile range 3.0-6.0 cups per day).

Our findings are consistent with the inverse association between coffee consumption and risk of diagnosed type 2 diabetes reported by cohort studies performed in a diverse variety of countries $[7,10,11,12$, 13]. In addition, our results are in agreement with the observed association between higher coffee consumption and lower prevalences of hyperglycaemia in Swedish [41], Japanese [42] and Dutch [43] populations. Conversely, in Pima Indians [9] and in a Finnish cohort [8] coffee consumption was not associated with type 2 diabetes. However, the study on Pima Indians only examined coffee consumption over a narrow range of values: the highest category of coffee consumption was 3 cups of coffee or more per day [9]. In addition, possible confounding by lifestyle factors was not controlled for in this study, which may have masked an existing association, since high coffee consumption has previously been shown to cluster with unhealthy behaviour (e.g. smoking, lack of physical activity, poor diet) in US populations [11]. The lack of an association in the Finnish study [8] may have been due to changing habits of coffee consumption in Finland during the long follow-up period or the higher consumption of boiled as opposed to filter coffee [12]. In the Netherlands, coffee was predominantly paper filtered [44]. In the present study, the association be- 
tween coffee consumption and type 2 diabetes was weaker than the association between coffee consumption and IGT. This may reflect a difference in the classification of type 2 diabetes and IGT. The diagnosis of type 2 diabetes is based on both fasting and post-load glucose concentrations, whereas IGT is based on postload glucose concentrations alone. In our study, postload concentrations of glucose were more strongly associated with coffee consumption than fasting levels. Alternatively, coffee consumption may have a more pronounced effect on the early stages of the development of type 2 diabetes than on the transition from IGT to type 2 diabetes. In healthy volunteers, consumption of decaffeinated coffee was associated with higher glucagon-like peptide-1 secretion following the oral administration of $25 \mathrm{~g}$ glucose [45]. Gut hormones such as glucagon-like peptide- 1 affect responses to oral glucose loads [46], and it could be speculated that these incretins contribute to the pronounced effect of coffee on post-load glucose concentrations suggested by our study.

Our findings suggest that habitual coffee consumption is associated with increased insulin sensitivity. This is consistent with the results from a study of 1088 Swedish elderly men [47] in which habitual coffee consumption was associated with increased insulin sensitivity as measured using a hyperinsulinaemiceuglycaemic clamp, but not with early insulin response during the OGTT (30-min increment of insulin per 30-min increment of glucose) [47]. In another Swedish study, coffee consumption was also associated with a lower HOMA-IR in women, but not in men [41]. In contrast to these findings, short-term metabolic studies have reported that caffeine acutely lowers insulin sensitivity $[18,19,20,21,22]$. However, the effects of long-term habitual coffee consumption on insulin sensitivity may be different from the acute effects of caffeine. Firstly, caffeine intake acutely increases blood pressure and concentrations of catecholamines, but these effects are attenuated after prolonged use of caffeine [48]. It is plausible that the adverse effect of caffeine on insulin sensitivity is also attenuated during chronic use [19]. Secondly, beneficial effects of caffeine and other constituents of coffee on insulin sensitivity have also been suggested [17, 28], and may only become apparent after prolonged use of coffee. Longer-term intervention studies examining the effect of caffeine and coffee consumption on insulin sensitivity are needed to explain the discrepancy between the findings from cohort studies and shortterm metabolic trials.

We were not able to analyse the effects of coffee and caffeine intake independently of each other, because consumption of other sources of caffeine and decaffeinated coffee [7] was relatively low in older Dutch populations at the time of the study. In a Japanese study, the inverse association between coffee consumption and glucose intolerance was stronger for coffee than for caffeine intake [42]. In two US cohorts, the inverse association between coffee consumption and the risk of type 2 diabetes could only be partly explained by caffeine intake [11]. These results suggest that coffee components other than caffeine may also affect glucose metabolism. Coffee contains many bioactive substances, including magnesium and the phenol chlorogenic acid. Our findings suggest that magnesium intake does not explain a substantial part of the association between coffee consumption and glucose intolerance. Chlorogenic acid is partly absorbed by the body [49], and metabolic effects in humans have been shown [50]. In a study in streptozotocin diabetic rats, oral administration of chlorogenic acid reduced plasma glucose levels over a 3 -h period [51]. Chlorogenic acid may reduce hepatic glucose output through inhibition of glucose-6-phosphatase activity [15], may attenuate glucose absorption rates by inhibiting the sodium-dependent glucose transporters [14], and may reduce oxidative stress through its anti-oxidant properties [14]. Roasting of coffee transforms some chlorogenic acid into quinides, compounds that have been shown to improve insulin sensitivity in rats [17].

In the present study, tea consumption was inversely associated with fasting glucose concentrations, but was not substantially associated with other parameters of glucose metabolism or the incidence of glucose intolerance. These results should be interpreted with caution, because the effect of tea consumption on glucose metabolism was not the primary hypothesis of our study. Also, in contrast with coffee consumption, tea consumption tends to be associated with a more health-conscious lifestyle, which increases the probability of confounding. Habitual tea consumption was shown to be associated with increased insulin sensitivity in a cross-sectional study [47], but was not associated with the risk of diagnosed type 2 diabetes in previous cohort studies [11, 12].

In conclusion, the results of the present study are in agreement with previous studies on diagnosed diabetes reporting a relationship between habitual coffee consumption and a lower incidence of glucose intolerance $[7,10,11,12,13]$. Our findings were consistent with a beneficial effect of habitual coffee consumption on insulin sensitivity, and suggest that coffee consumption affects post-load rather than fasting glucose metabolism. It is premature to advocate high coffee consumption as a means of reducing the risk of type 2 diabetes. However, the available data on coffee consumption and glucose metabolism warrant mechanistic studies of coffee constituents and longer-term randomised trials investigating the effects of coffee on insulin sensitivity and glucose tolerance. 


\section{References}

1. Tavani A, La Vecchia C (2000) Coffee and cancer: a review of epidemiological studies, 1990-1999. Eur J Cancer Prev 9:241-256

2. Jee SH, He J, Appel LJ, Whelton PK, Suh I, Klag MJ (2001) Coffee consumption and serum lipids: a meta-analysis of randomized controlled clinical trials. Am J Epidemiol 153:353-362

3. Jee SH, He J, Whelton PK, Suh I, Klag MJ (1999) The effect of chronic coffee drinking on blood pressure: a metaanalysis of controlled clinical trials. Hypertension 33:647-652

4. Kawachi I, Colditz GA, Stone CB (1994) Does coffee drinking increase the risk of coronary heart disease? Results from a meta-analysis. Br Heart J 72:269-275

5. Leitzmann MF, Stampfer MJ, Willett WC, Spiegelman D, Colditz GA, Giovannucci EL (2002) Coffee intake is associated with lower risk of symptomatic gallstone disease in women. Gastroenterology 123:1823-1830

6. Heaney RP (2002) Effects of caffeine on bone and the calcium economy. Food Chem Toxicol 40:1263-1270

7. van Dam RM, Feskens EJ (2002) Coffee consumption and risk of type 2 diabetes mellitus. Lancet 360:1477-1478

8. Reunanen A, Heliovaara M, Aho K (2003) Coffee consumption and risk of type 2 diabetes mellitus. Lancet 361:702-703

9. Saremi A, Tulloch-Reid M, Knowler WC (2003) Coffee consumption and the incidence of type 2 diabetes. Diabetes Care 26:2211-2212

10. Rosengren A, Dotevall A, Wilhelmsen L, Thelle D, Johansson S (2004) Coffee and incidence of diabetes in Swedish women: a prospective 18-year follow-up study. J Intern Med 255:89-95

11. Salazar-Martinez E, Willett WC, Ascherio A et al. (2004) Coffee consumption and risk for type 2 diabetes mellitus. Ann Intern Med 140:1-8

12. Tuomilehto J, Hu G, Bidel S, Lindstrom J, Jousilahti $P$ (2004) Coffee consumption and risk of type 2 diabetes mellitus among middle-aged Finnish men and women. JAMA 291:1213-1219

13. Carlsson S, Hammar N, Grill V, Kaprio J (2004) Coffee consumption and risk of type 2 diabetes in Finnish twins. Int J Epidemiol 33:616-617

14. Clifford MN (2000) Chlorogenic acid and other cinnamates-nature, occurrence, dietary burden, absorption and metabolism. J Sci Food Agric 80:1033-1043

15. Arion WJ, Canfield WK, Ramos FC et al. (1997) Chlorogenic acid and hydroxynitrobenzaldehyde: new inhibitors of hepatic glucose-6-phosphatase. Arch Biochem Biophys 339:315-322

16. Herling AW, Burger H, Schubert G, Hemmerle H, Schaefer H, Kramer W (1999) Alterations of carbohydrate and lipid intermediary metabolism during inhibition of glucose-6phosphatase in rats. Eur J Pharmacol 386:75-82

17. Shearer J, Farah A, de Paulis T et al. (2003) Quinides of roasted coffee enhance insulin action in conscious rats. J Nutr 133:3529-3532

18. Greer F, Hudson R, Ross R, Graham T (2001) Caffeine ingestion decreases glucose disposal during a hyperinsulinemic-euglycemic clamp in sedentary humans. Diabetes 50:2349-2354

19. Keijzers GB, De Galan BE, Tack CJ, Smits P (2002) Caffeine can decrease insulin sensitivity in humans. Diabetes Care 25:364-369

20. Thong FS, Derave W, Kiens B et al. (2002) Caffeine-induced impairment of insulin action but not insulin signal- ing in human skeletal muscle is reduced by exercise. Diabetes $51: 583-590$

21. Thong FS, Graham TE (2002) Caffeine-induced impairment of glucose tolerance is abolished by beta-adrenergic receptor blockade in humans. J Appl Physiol 92: 2347-2352

22. Petrie HJ, Chown SE, Belfie LM et al. (2004) Caffeine ingestion increases the insulin response to an oral-glucosetolerance test in obese men before and after weight loss. Am J Clin Nutr 80:22-28

23. Cheraskin E, Ringsdorf WM Jr., Setyaadmadja AT, Barrett RA (1967) Effect of caffeine versus placebo supplementation on blood-glucose concentration. Lancet 1:1299-1300

24. Jankelson OM, Beaser SB, Howard FM, Mayer J (1967) Effect of coffee on glucose tolerance and circulating insulin in men with maturity-onset diabetes. Lancet 1:527-529

25. Wachman A, Hattner RS, George B, Bernstein DS (1970) Effects of decaffeinated and nondecaffeinated coffee ingestion on blood glucose and plasma radioimmunoreactive insulin responses to rapid intravenous infusion of glucose in normal man. Metabolism 19:539-546

26. Pizziol A, Tikhonoff V, Paleari CD et al. (1998) Effects of caffeine on glucose tolerance: a placebo-controlled study. Eur J Clin Nutr 52:846-849

27. Lane JD, Barkauskas CE, Surwit RS, Feinglos MN (2004) Caffeine impairs glucose metabolism in type 2 diabetes. Diabetes Care 27:2047-2048

28. Isogawa A, Noda M, Takahashi Y, Kadowaki T, Tsugane S (2003) Coffee consumption and risk of type 2 diabetes mellitus. Lancet 361:703-704

29. Bruton JD, Lemmens R, Shi CL et al. (2003) Ryanodine receptors of pancreatic beta-cells mediate a distinct contextdependent signal for insulin secretion. FASEB J 17:301-303

30. Salmeron J, Manson JE, Stampfer MJ, Colditz GA, Wing AL, Willett WC (1997) Dietary fiber, glycemic load, and risk of non-insulin-dependent diabetes mellitus in women. JAMA 277:472-477

31. Salmeron J, Ascherio A, Rimm EB et al. (1997) Dietary fiber, glycemic load, and risk of NIDDM in men. Diabetes Care 20:545-550

32. Meyer KA, Kushi LH, Jacobs DRJ, Slavin J, Sellers TA, Folsom AR (2000) Carbohydrates, dietary fiber, and incident type 2 diabetes in older women. Am J Clin Nutr 71:921-930

33. Paolisso G, Sgambato S, Gambardella A, et al. (1992) Daily magnesium supplements improve glucose handling in elderly subjects. Am J Clin Nutr 55:1161-1167

34. Mooy JM, Grootenhuis PA, de Vries H et al. (1996) Intraindividual variation of glucose, specific insulin and proinsulin concentrations measured by two oral glucose tolerance tests in a general Caucasian population: the Hoorn Study. Diabetologia 39:298-305

35. Matthews DR, Hosker JP, Rudenski AS, Naylor BA, Treacher DF, Turner RC (1985) Homeostasis model assessment: insulin resistance and beta-cell function from fasting plasma glucose and insulin concentrations in man. Diabetologia 28:412-419

36. World Health Organization (1999) Definition, diagnosis and classification of diabetes mellitus and its complications. Report of a WHO Consultation. World Health Organization, Geneva

37. Grootenhuis PA, Westenbrink S, Sie CM, de Neeling JN, Kok FJ, Bouter LM (1995) A semiquantitative food frequency questionnaire for use in epidemiologic research among the elderly: validation by comparison with dietary history. J Clin Epidemiol 48:859-868 
38. Caspersen CJ, Bloemberg BP, Saris WH, Merritt RK, Kromhout D (1991) The prevalence of selected physical activities and their relation with coronary heart disease risk factors in elderly men: the Zutphen Study, 1985. Am J Epidemiol 133:1078-1092

39. Rose GA, Blackburn H (1982) Cardiovascular survey methods. World Health Organization, Geneva

40. Willett WC, Stampfer MJ (1998) Implications of total energy intake for epidemiological analysis. In: Willett WC (ed) Nutritional epidemiology, 2nd edn. Oxford University Press, New York, pp 273-301

41. Agardh EE, Carlsson S, Ahlbom A, Efendic S, Grill V, Hammar $\mathrm{N}$ et al. (2003) Coffee consumption, type 2 diabetes and impaired glucose tolerance in Swedish men and women. J Intern Med 255:645-652

42. Yoshioka K, Kogure A, Yoshida T, Yoshikawa T (2002) Coffee consumption and risk of type 2 diabetes mellitus. Lancet 361:703

43. van Dam RM, Feskens EJ, Kromhout D (2003) Coffee consumption in relation to hyperinsulinemia and glucose tolerance in elderly men. Ann Nutr Metab 47:627-628 (Abstract)

44. Urgert R, de Groot CP (1996) Consumption of unfiltered coffee brews in elderly Europeans. SENECA Investigators. Eur J Clin Nutr 50 [Suppl 2]:S101-S104
45. Johnston KL, Clifford MN, Morgan LM (2003) Coffee acutely modifies gastrointestinal hormone secretion and glucose tolerance in humans: glycemic effects of chlorogenic acid and caffeine. Am J Clin Nutr 78:728-733

46. Drucker DJ. Glucagon-like peptides (1998) Diabetes 47:159-169

47. Arnlov J, Vessby B, Riserus U (2004) Coffee consumption and insulin sensitivity. JAMA 291:1199-1201

48. Denaro CP, Brown CR, Jacob P 3rd, Benowitz NL (1991) Effects of caffeine with repeated dosing. Eur J Clin Pharmacol 40:273-278

49. Olthof MR, Hollman PC, Katan MB (2001) Chlorogenic acid and caffeic acid are absorbed in humans. J Nutr 131:66-71

50. Olthof MR, Hollman PC, Zock PL, Katan MB (2001) Consumption of high doses of chlorogenic acid, present in coffee, or of black tea increases plasma total homocysteine concentrations in humans. Am J Clin Nutr 73:532-538

51. Andrade-Cetto A, Wiedenfeld H (2001) Hypoglycemic effect of Cecropia obtusifolia on streptozotocin diabetic rats. J Ethnopharmacol 78:145-149 